Canadian

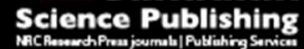

Canadian Journal of Chemistry Revue canadienne de chimie

\title{
On Topological Properties of Dominating David Derived Networks
}

\begin{tabular}{|r|l|}
\hline Journal: & Canadian Journal of Chemistry \\
\hline Manuscript ID & cjc-2015-0185.R1 \\
\hline Manuscript Type: & Article \\
\hline Date Submitted by the Author: & $29-$ Sep-2015 \\
\hline Complete List of Authors: & $\begin{array}{l}\text { Imran, Muhammad; National University of Sciences \& Technology (NUST), } \\
\text { School of Natural Sciences } \\
\text { Baig, A. Q.; COMSATS Insitute of Information Technology, Attock, } \\
\text { Pakistan, Mathematics } \\
\text { Ali, Haidar; COMSATS Insitute of Information Technology, Attock, Pakistan, } \\
\text { Mathematics }\end{array}$ \\
\hline Keyword: & $\begin{array}{l}\text { General Randi\'\{c\} index, Atom-bond connectivity \$(ABC)\$ index, } \\
\text { Geometric-arithmetic \$(GA)\$ index, Star of David, Dominating David } \\
\text { Derived network }\end{array}$ \\
\hline
\end{tabular}




\title{
On Topological Properties of Dominating David Derived Networks ${ }^{\star}$
}

\author{
Muhammad Imran*, Abdul Qudair Baig ${ }^{1}, \mathrm{Haidar} \mathrm{Ali}^{2}$ \\ *Department of Mathematics, \\ School of Natural Sciences (SNS), \\ National University of Sciences and Technology (NUST), \\ Sector H-12, Islamabad, Pakistan \\ Tel: +923334736997 \\ Email: imrandhab@gmail.com \\ ${ }^{1}$ Department of Mathematics, \\ COMSATS Institute of Information Technology, Attock \\ ${ }^{2}$ Department of Mathematics, \\ Government College University, Faisalabad \\ \{aqbaig1, haidar3830\}@gmail.com
}

* This research is supported by COMSATS Attock and NUST, Islamabad, Pakistan and by the grant of Higher Education Commission of Pakistan Ref. No. 20-367/NRPU/R\&D/HEC/12/831. 
Abstract. Topological indices are numerical parameters of a graph which characterize its molecular topology and are usually graph invariant. In QSAR/QSPR study, physico-chemical properties and topological indices such as Randić, atom-bond connectivity $(A B C)$ and geometric-arithmetic $(G A)$ index are used to predict the bioactivity of chemical compounds. Graph theory has found a considerable use in this important area of research.

All the studied interconnection networks in this paper are constructed by the Star of David network. In this paper, we study the general Randić, first Zagreb, $A B C, G A, A B C_{4}$ and $G A_{5}$ indices for the first, second and third type of Dominating David Derived networks and give closed formulas of these indices for these networks. These results are useful in network science to understand the underlying topologies of these networks.

2010 Mathematics Subject Classification: 05C12, 05C90

Keywords: General Randić index, Atom-bond connectivity $(A B C)$ index, Geometric-arithmetic $(G A)$ index, Star of David, Dominating David Derived network. 


\section{Introduction and preliminary results}

Graph theory has provided chemists with a variety of useful tools, such as topological indices. Molecules and molecular compounds are often modeled by a molecular graph. A molecular graph is a representation of the structural formula of a chemical compound in terms of graph theory, whose vertices correspond to the atoms of the compound and edges correspond to chemical bonds. Cheminformatics is new subject which is a combination of chemistry, mathematics and information science. It studies Quantitative structure-activity (QSAR) and structure-property (QSPR) relationships that are used to predict the biological activities and properties of the chemical compounds. In the QSAR /QSPR study, physicochemical properties and topological indices such as Wiener index, Szeged index, Randić index, Zagreb indices and $A B C$ index are used to predict bioactivity of the chemical compounds.

A graph can be recognized by a numeric number, a polynomial, a sequence of numbers or by a matrix. A topological index is a numeric quantity associated with a graph which characterize the topology of graph and is invariant under graph automorphism. There are some major classes of topological indices such as distance based topological indices, degree based topological indices and counting related polynomials and indices of graphs. Among these classes degree based topological indices are of great importance and play a vital role in chemical graph theory and particularly in theoretical chemistry. In more precise way, a topological index $\operatorname{Top}(G)$ of a graph, is a number with the property that for every graph $H$ isomorphic to $G$, we have $\operatorname{Top}(H)=\operatorname{Top}(G)$. The concept of topological indices came from the work done by Wiener ${ }^{1}$ while he was working on the boiling point of paraffin (an important member of alkane family). He named this index as path number. Later on, the path number was renamed as Wiener index ${ }^{2}$ and the whole theory of topological indices started.

The algorithm for constructing David Derived and Dominating David Derived network (of dimension $n)$ is as follows: Consider a Star of David network $S D(n)^{3}$ of dimension $n$. Split each edge of it into two by inserting a new vertex. Resulting graph is called David Derived network $D D(n)$ of dimension $n$. Consider a honeycomb network denoted by $H C(n)$ of dimension $n$. Split each edge of $H C(n)$ into two by inserting a new vertex. In each hexagon cell, connect the new vertices by an edge if they are at a distance of 4 units within the cell. Place vertices at new edge crossings. Remove the initial vertices and edges of $H C(n)$. Split each horizontal edge into two edges by inserting a new vertex. Resulting graph is called Dominating David Derived network $D D D(n)$ of dimension $n$.

The Dominating David Derived network of first type denoted by $D_{1}(n)$ of dimension $n$ can be obtained by connecting vertices of degree two of $D D D(n)$ by an edge, which are not in the boundary. The Dominating David Derived network of second type denoted by $D_{2}(n)$ of dimension $n$ can be obtained by subdividing once the new edge introduced in $D_{1}(n)$. The Dominating David Derived network of third type $D_{3}(n)$ of dimension $n$ can be obtained from $D_{1}(n)$ by introducing parallel path of length 2 between the vertices of degree two which are not in the boundary ${ }^{4}$. 
In this article, $G$ is considered to be a network with vertex set $V(G)$ and edge set $E(G)$, the $\operatorname{deg}(u)$ is the degree of vertex $u \in V(G)$ and $S_{u}=\sum_{v \in N_{G}(u)} \operatorname{deg}(v)$ where $N_{G}(u)=\{v \in V(G) \mid u v \in E(G)\}$. The notations used in this article are mainly taken from the books ${ }^{5,6}$.

Let $G$ be a connected graph. Then the Wiener index of $G$ is defined as

$$
W(G)=\frac{1}{2} \sum_{(u, v)} d(u, v)
$$

where $(u, v)$ is any ordered pair of vertices in $G$ and $d(u, v)$ is $u-v$ geodesic.

The very first and oldest degree based topological index is Randić index ${ }^{7}$ denoted by $R_{-\frac{1}{2}}(G)$ and was introduced by Milan Randić and is defined as

$$
R_{-\frac{1}{2}}(G)=\sum_{u v \in E(G)} \frac{1}{\sqrt{\operatorname{deg}(u) \operatorname{deg}(v)}}
$$

The general Randić index $R_{\alpha}(G)$ is the sum of $(\operatorname{deg}(u) \operatorname{deg}(v))^{\alpha}$ over all the edges $e=u v \in E(G)$ and is defined as

$$
R_{\alpha}(G)=\sum_{u v \in E(G)}(\operatorname{deg}(u) \operatorname{deg}(v))^{\alpha} \text { for } \alpha=1, \frac{1}{2},-1,-\frac{1}{2}
$$

An important topological index introduced by Ivan Gutman and Trinajstić is the Zagreb index denoted by $M_{1}(G)$ and is defined as

$$
M_{1}(G)=\sum_{u v \in E(G)}(\operatorname{deg}(u)+\operatorname{deg}(v))
$$

One of the well-known degree based topological index is atom-bond connectivity $(A B C)$ index introduced by Estrada et $a l .^{8}$ and defined as

$$
A B C(G)=\sum_{u v \in E(G)} \sqrt{\frac{\operatorname{deg}(u)+\operatorname{deg}(v)-2}{\operatorname{deg}(u) \operatorname{deg}(v)}}
$$

Another well-known connectivity topological descriptor is geometric-arithmetic $(G A)$ index which was introduced by Vukičević et $a l .^{9}$ and defined as

$$
G A(G)=\sum_{u v \in E(G)} \frac{2 \sqrt{\operatorname{deg}(u) \operatorname{deg}(v)}}{\operatorname{deg}(u)+\operatorname{deg}(v)}
$$

The fourth version of atom-bond connectivity index denoted by $A B C_{4}$ and fifth version of geometricarithmetic index denoted by $G A_{5}$ can be computed if we are able to find the edge partition of these interconnection networks based on sum of the degrees of end vertices of each edge in these graphs. The 
fourth version of $A B C$ index is introduced by Ghorbani et al. ${ }^{10}$ and defined as

$$
A B C_{4}(G)=\sum_{u v \in E(G)} \sqrt{\frac{S_{u}+S_{v}-2}{S_{u} S_{v}}}
$$

Recently, the fifth version of $G A$ index is proposed by Graovac et al. ${ }^{11}$ and is defined as

$$
G A_{5}(G)=\sum_{u v \in E(G)} \frac{2 \sqrt{S_{u} S_{v}}}{S_{u}+S_{v}}
$$

The general Randić index for $\alpha=1$ is the second Zagreb index for any graph $G$.

\section{Main results}

In this paper, we study the general Randić, first Zagreb, $A B C, G A, A B C_{4}$ and $G A_{5}$ indices and give closed formulas of these indices for the first, second and third type of Dominating David Derived network. Hayat et al. studied recently the degree based topological indices for various networks like silicates, hexagonal, honeycomb and oxide ${ }^{12}$. Nowadays there is an extensive research activity on $A B C$ and $G A$ indices and their variants. For further study of topological indices of various graph families and their invariants see, ${ }^{13-23}$.

\subsection{Results for Dominating David Derived networks of first type}

In this section, we compute certain degree based topological indices of Dominating David Derived network of first type denoted by $D_{1}(n)$. We compute the general Randić $R_{\alpha}(G)$ for $\alpha=\left\{1,-1, \frac{1}{2},-\frac{1}{2}\right\}, A B C$, $G A, A B C_{4}$ and $G A_{5}$ indices for first type of Dominating David Derived networks in this section.

In the following theorem, the general Randić index for Dominating David Derived network of first type is computed.

Theorem 2.1.1. Let $G \cong D_{1}(n)$ be the Dominating David Derived network of first type, then its general Randić index is equal to

$$
R_{\alpha}\left(D_{1}(n)\right)= \begin{cases}1089 n^{2}-1357 n+501, & \alpha=1 ; \\ (72 \sqrt{3}+171) n^{2}+(56 \sqrt{2}-112 \sqrt{3}+4 \sqrt{6}-239) n- & \\ 32 \sqrt{2}+48 \sqrt{3}-4 \sqrt{6}+95, & \alpha=\frac{1}{2} ; \\ \frac{1}{4}\left(25 n^{2}-\frac{151}{9} n+\frac{41}{9}\right), & \alpha=-1 ; \\ (6 \sqrt{3}+12) n^{2}+\left(7 \sqrt{2}-\frac{28}{3} \sqrt{3}+\frac{2}{3} \sqrt{6}-\frac{46}{3}\right) n- & \\ 4 \sqrt{2}+4 \sqrt{3}-\frac{2}{3} \sqrt{6}+\frac{20}{3}, & \alpha=-\frac{1}{2} .\end{cases}
$$




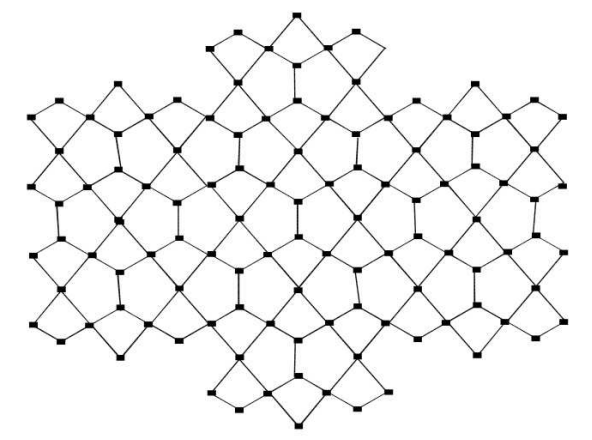

Fig. 1. Dominating David Derived network of first type $\left(D_{1}(2)\right)$

\begin{tabular}{|c|c|}
\hline$\left(d_{u}, d_{v}\right)$ where $u v \in E(G)$ & Number of edges \\
\hline$(2,2)$ & $4 n$ \\
\hline$(2,3)$ & $4 n-4$ \\
\hline$(2,4)$ & $28 n-16$ \\
\hline$(3,3)$ & $9 n^{2}-13 n+5$ \\
\hline$(3,4)$ & $36 n^{2}-56 n+24$ \\
\hline$(4,4)$ & $36 n^{2}-52 n+20$ \\
\hline
\end{tabular}

Table 1. Edge partition of Dominating David Derived network of first type $\left(D_{1}(n)\right)$ based on degrees of end vertices of each edge.

Proof. Let $G$ be the Dominating David Derived network of first type. The Dominating David Derived network of first type $\left(D_{1}(n)\right)$ has $20 n-10$ vertices of degree $2,18 n^{2}-26 n+10$ vertices of degree 3 and $27 n^{2}-33 n+12$ vertices of degree 4 . The edge set of $D_{1}(n)$ can be divided into six partitions based on the degree of end vertices. The first edge partition $E_{1}\left(D_{1}(n)\right)$ contains $4 n$ edges $u v$; where $\operatorname{deg}(u)=\operatorname{deg}(v)=$ 2. The second edge partition $E_{2}\left(D_{1}(n)\right)$ contains $4 n-4$ edges $u v$, where $\operatorname{deg}(u)=2$ and $\operatorname{deg}(v)=3$. The third edge partition $E_{3}\left(D_{1}(n)\right)$ contains $28 n-16$ edges $u v$; where $\operatorname{deg}(u)=2$ and $\operatorname{deg}(v)=4$. The fourth edge partition $E_{4}\left(D_{1}(n)\right)$ contains $9 n^{2}-13 n+5$ edges $u v$; where $\operatorname{deg}(u)=\operatorname{deg}(v)=3$. The fifth edge partition $E_{5}\left(D_{1}(n)\right)$ contains $36 n^{2}-56 n+24$ edges $u v$; where we have $\operatorname{deg}(u)=3$ and $\operatorname{deg}(v)=4$. The sixth edge partition $E_{6}\left(D_{1}(n)\right)$ contains $36 n^{2}-52 n+20$ edges $u v ;$ where $\operatorname{deg}(u)=\operatorname{deg}(v)=4$. Table 1 shows such an edge partition of $D_{1}(n)$. Thus from equation (3), is follows that

$$
R_{\alpha}(G)=\sum_{u v \in E(G)}(\operatorname{deg}(u) \cdot \operatorname{deg}(v))^{\alpha}
$$

For $\alpha=1$

Now we apply the formula of general Randić index $R_{\alpha}(G)$ for $\alpha=1$.

$$
R_{1}(G)=\sum_{j=1}^{6} \sum_{u v \in E_{j}(G)} \operatorname{deg}(u) \cdot \operatorname{deg}(v)
$$


By using the edge partition given in Table 1, we get the following

$$
\begin{gathered}
R_{1}(G)=4\left|E_{1}\left(D_{1}(n)\right)\right|+6\left|E_{2}\left(D_{1}(n)\right)\right|+8\left|E_{3}\left(D_{1}(n)\right)\right|+9\left|E_{4}\left(D_{1}(n)\right)\right|+12\left|E_{5}\left(D_{1}(n)\right)\right|+16\left|E_{6}\left(D_{1}(n)\right)\right| \\
\Longrightarrow R_{1}(G)=1089 n^{2}-1357 n+501
\end{gathered}
$$

For $\alpha=\frac{1}{2}$

We apply the formula of $R_{\alpha}(G)$ for $\alpha=\frac{1}{2}$.

$$
R_{\frac{1}{2}}(G)=\sum_{j=1}^{6} \sum_{u v \in E_{j}(G)} \sqrt{\operatorname{deg}(u) \cdot \operatorname{deg}(v)}
$$

By using the edge partition given in Table 1, we get

$$
\begin{aligned}
& R_{\frac{1}{2}}(G)=2\left|E_{1}\left(D_{1}(n)\right)\right|+\sqrt{6}\left|E_{2}\left(D_{1}(n)\right)\right|+2 \sqrt{2}\left|E_{3}\left(D_{1}(n)\right)\right|+3\left|E_{4}\left(D_{1}(n)\right)\right|+2 \sqrt{3}\left|E_{5}\left(D_{1}(n)\right)\right|+4\left|E_{6}\left(D_{1}(n)\right)\right| \\
& \Longrightarrow R_{\frac{1}{2}}(G)=(72 \sqrt{3}+171) n^{2}+(56 \sqrt{2}-112 \sqrt{3}+4 \sqrt{6}-239) n-32 \sqrt{2}+48 \sqrt{3}-4 \sqrt{6}+95
\end{aligned}
$$

For $\alpha=-1$

We apply the formula of $R_{\alpha}(G)$ for $\alpha=-1$.

$$
R_{-1}(G)=\sum_{j=1}^{6} \sum_{u v \in E_{j}(G)} \frac{1}{\operatorname{deg}(u) \cdot \operatorname{deg}(v)}
$$

$R_{-1}(G)=\frac{1}{4}\left|E_{1}\left(D_{1}(n)\right)\right|+\frac{1}{6}\left|E_{2}\left(D_{1}(n)\right)\right|+\frac{1}{8}\left|E_{3}\left(D_{1}(n)\right)\right|+\frac{1}{9}\left|E_{4}\left(D_{1}(n)\right)\right|+\frac{1}{12}\left|E_{5}\left(D_{1}(n)\right)\right|+\frac{1}{16}\left|E_{6}\left(D_{1}(n)\right)\right|$

$$
\Longrightarrow R_{-1}(G)=\frac{1}{4}\left(25 n^{2}-\frac{151}{9} n+\frac{41}{9}\right)
$$

For $\alpha=-\frac{1}{2}$

We apply the formula of $R_{\alpha}(G)$ for $\alpha=-\frac{1}{2}$.

$$
\begin{gathered}
R_{-\frac{1}{2}}(G)=\sum_{j=1}^{6} \sum_{u v \in E_{j}(G)} \frac{1}{\sqrt{\operatorname{deg}(u) \cdot \operatorname{deg}(v)}} \\
R_{-\frac{1}{2}}(G)=\frac{1}{2}\left|E_{1}\left(D_{1}(n)\right)\right|+\frac{1}{\sqrt{6}}\left|E_{2}\left(D_{1}(n)\right)\right|+\frac{\sqrt{2}}{4}\left|E_{3}\left(D_{1}(n)\right)\right|+\frac{1}{3}\left|E_{4}\left(D_{1}(n)\right)\right|+\frac{\sqrt{3}}{6}\left|E_{5}\left(D_{1}(n)\right)\right|+\frac{1}{4}\left|E_{6}\left(D_{1}(n)\right)\right| \\
\Longrightarrow R_{-\frac{1}{2}}(G)=(6 \sqrt{3}+12) n^{2}+\left(7 \sqrt{2}-\frac{28}{3} \sqrt{3}+\frac{2}{3} \sqrt{6}-\frac{46}{3}\right) n-4 \sqrt{2}+4 \sqrt{3}-\frac{2}{3} \sqrt{6}+\frac{20}{3}
\end{gathered}
$$

In the following theorem, we compute the first Zagreb index for Dominating David Derived network of first type denoted by $D_{1}(n)$. 
Theorem 2.1.2. For Dominating David Derived network of first type $\left(G \cong D_{1}(n)\right.$, the first Zagreb index is equal to

$$
M_{1}\left(D_{1}(n)\right)=594 n^{2}-682 n+242
$$

Proof. Let $G$ be the Dominating David Derived network of first type $\left(D_{1}(n)\right)$. By using the edge partition from Table 1, the result directly follows. From the equation (4), we have

$$
\begin{gathered}
M_{1}(G)=\sum_{u v \in E(G)}(\operatorname{deg}(u)+\operatorname{deg}(v))=\sum_{j=1}^{6} \sum_{u v \in E_{j}(G)}(\operatorname{deg}(u)+\operatorname{deg}(v)) \\
M_{1}(G)=4\left|E_{1}\left(D_{1}(n)\right)\right|+5\left|E_{2}\left(D_{1}(n)\right)\right|+6\left|E_{3}\left(D_{1}(n)\right)\right|+6\left|E_{4}\left(D_{1}(n)\right)\right|+7\left|E_{5}\left(D_{1}(n)\right)\right|+8\left|E_{6}\left(D_{1}(n)\right)\right| .
\end{gathered}
$$

By doing some elementary calculation, we get

$$
\Longrightarrow M_{1}(G)=594 n^{2}-682 n+242
$$

In the next theorem, we compute the $A B C, G A, A B C_{4}$ and $G A_{5}$ indices of Dominating David Derived network of first type denoted by $D_{1}(n)$.

Theorem 2.1.3. Let $G \cong D_{1}(n)$ be the Dominating David Derived network of first type for every positive integer $n \geq 2$; then we have

- $A B C\left(D_{1}(n)\right)=(6+9 \sqrt{6}+6 \sqrt{15}) n^{2}+\left(\frac{54 \sqrt{2}-39 \sqrt{6}-28 \sqrt{15}-26}{3}\right) n+\left(\frac{10-30 \sqrt{2}+12 \sqrt{15}+15 \sqrt{6}}{3}\right)$.

- $G A\left(D_{1}(n)\right)=\left(\frac{315+144 \sqrt{3}}{7}\right) n^{2}+\left(\frac{280 \sqrt{2}-480 \sqrt{3}+24 \sqrt{6}-915}{15}\right) n+\left(\frac{2625-1120 \sqrt{2}+1440 \sqrt{3}-168 \sqrt{6}}{105}\right)$.

- $A B C_{4}\left(D_{1}(n)\right)=\left(\frac{63 \sqrt{20}+693 \sqrt{2}+18 \sqrt{3542}}{77}\right) n^{2}+\left(\frac{8}{9}-\frac{53}{3} \sqrt{2}+\frac{2}{3} \sqrt{6}+\frac{2}{7} \sqrt{26}+\frac{4}{13} \sqrt{26}+\frac{3}{13} \sqrt{39}+\frac{4}{\sqrt{7}}+\frac{5}{\sqrt{11}}+\right.$ $\left.\frac{2}{3} \sqrt{10}+\frac{2}{11} \sqrt{110}+\frac{2}{7} \sqrt{42}+\frac{2}{21} \sqrt{357}+\frac{3}{11} \sqrt{374}+\frac{\sqrt{494}}{13}-\frac{7}{11} \sqrt{20}-\frac{34}{77} \sqrt{3542}+\frac{20}{\sqrt{182}}\right) n+10 \sqrt{2}-\frac{2}{3} \sqrt{6}-\frac{3}{13} \sqrt{39}-$ $\frac{2}{7} \sqrt{42}-\frac{4}{\sqrt{7}}-\frac{5}{\sqrt{11}}-\frac{4}{13} \sqrt{26}+\frac{2}{11} \sqrt{77}-\frac{2}{21} \sqrt{357}-\frac{2}{11} \sqrt{374}-\frac{\sqrt{494}}{13}+\frac{16}{77} \sqrt{3542}-\frac{20}{\sqrt{182}}-\frac{8}{9}+\frac{3}{11} \sqrt{20}-\frac{2}{7} \sqrt{26}$. - $G A_{5}\left(D_{1}(n)\right)=\left(\frac{225+240 \sqrt{14}+72 \sqrt{154}}{25}\right) n^{2}+\left(3+\frac{3}{2} \sqrt{7}+\frac{32}{27} \sqrt{11}+\frac{32}{29} \sqrt{13}-\frac{304}{15} \sqrt{14}+\frac{24}{23} \sqrt{14}+\frac{4}{5} \sqrt{21}+\right.$ $\left.\frac{16}{19} \sqrt{21}+\frac{48}{19} \sqrt{22}+\frac{16}{21} \sqrt{26}+\frac{8}{13} \sqrt{42}+\frac{8}{17} \sqrt{66}+\frac{\sqrt{143}}{3}+\frac{8}{27} \sqrt{182}-\frac{136}{25} \sqrt{154}\right) n+\frac{8}{3} \sqrt{2}-\frac{3}{2} \sqrt{7}-\frac{32}{27} \sqrt{11}-\frac{32}{29} \sqrt{13}+$ $\frac{32}{3} \sqrt{14}-\frac{24}{23} \sqrt{14}-\frac{4}{5} \sqrt{21}-\frac{16}{19} \sqrt{21}-\frac{32}{19} \sqrt{22}-\frac{16}{21} \sqrt{26}+\frac{16}{23} \sqrt{33}-\frac{8}{13} \sqrt{42}-\frac{\sqrt{143}}{3}+\frac{64}{25} \sqrt{154}-\frac{8}{27} \sqrt{182}-3$.

Proof. By finding the edge partition given in Table 1 and then by using the definition, we get the required result. From equation (5), it follows that

$$
A B C(G)=\sum_{u v \in E(G)} \sqrt{\frac{\operatorname{deg}(u)+\operatorname{deg}(v)-2}{\operatorname{deg}(u) \cdot \operatorname{deg}(v)}}=\sum_{j=1}^{6} \sum_{u v \in E_{j}(G)} \sqrt{\frac{\operatorname{deg}(u)+\operatorname{deg}(v)-2}{\operatorname{deg}(u) \cdot \operatorname{deg}(v)}}
$$

$A B C\left(D_{1}(n)\right)=\frac{1}{\sqrt{2}}\left|E_{1}\left(D_{1}(n)\right)\right|+\frac{1}{\sqrt{2}}\left|E_{2}\left(D_{1}(n)\right)\right|+\frac{1}{\sqrt{2}}\left|E_{3}\left(D_{1}(n)\right)\right|+\frac{2}{3}\left|E_{4}\left(D_{1}(n)\right)\right|+\frac{\sqrt{15}}{6}\left|E_{5}\left(D_{1}(n)\right)\right|+$ $\frac{\sqrt{6}}{4}\left|E_{6}\left(D_{1}(n)\right)\right|$. 
By doing the some calculation, we get

$\Longrightarrow A B C\left(D_{1}(n)\right)=(6+9 \sqrt{6}+6 \sqrt{15}) n^{2}+\left(\frac{54 \sqrt{2}-39 \sqrt{6}-28 \sqrt{15}-26}{3}\right) n+\left(\frac{10-30 \sqrt{2}+12 \sqrt{15}+15 \sqrt{6}}{3}\right)$.

From equation(6), we get

$$
G A\left(D_{1}(n)\right)=\sum_{u v \in E(G)} \frac{2 \sqrt{\operatorname{deg}(u) \cdot \operatorname{deg}(v)}}{\operatorname{deg}(u)+\operatorname{deg}(v)}=\sum_{j=1}^{6} \sum_{u v \in E_{j}(G)} \frac{2 \sqrt{\operatorname{deg}(u) \cdot \operatorname{deg}(v)}}{\operatorname{deg}(u)+\operatorname{deg}(v)}
$$

By doing some calculation, we get

$$
\begin{aligned}
& G A\left(D_{1}(n)\right)=\left|E_{1}\left(D_{1}(n)\right)\right|+2 \frac{\sqrt{6}}{5}\left|E_{2}\left(D_{1}(n)\right)\right|+2 \frac{\sqrt{2}}{3}\left|E_{3}\left(D_{1}(n)\right)\right|+\left|E_{4}\left(D_{1}(n)\right)\right|+4 \frac{\sqrt{3}}{7}\left|E_{5}\left(D_{1}(n)\right)\right|+ \\
& \left|E_{6}\left(D_{1}(n)\right)\right| \\
& \Longrightarrow G A\left(D_{1}(n)\right)=\left(\frac{315+144 \sqrt{3}}{7}\right) n^{2}+\left(\frac{280 \sqrt{2}-480 \sqrt{3}+24 \sqrt{6}-915}{15}\right) n+\left(\frac{2625-1120 \sqrt{2}+1440 \sqrt{3}-168 \sqrt{6}}{105}\right) .
\end{aligned}
$$

If we consider edge partitions based on degree sum of neighbors of end vertices; then the edge set $E\left(D_{1}(n)\right)$ can be divided into twenty edge partition $E_{j}\left(D_{1}(n)\right), 7 \leq j \leq 26$, where the edge partition $E_{7}\left(D_{1}(n)\right)$ contains $4 n$ edges $u v$ with $S_{u}=S_{v}=6$; the edge partition $E_{8}\left(D_{1}(n)\right)$ contains $4 n$ edges $u v$ with $S_{u}=6$ and $S_{v}=11$; the edge partition $E_{9}\left(D_{1}(n)\right)$ contains 4 edges $u v$ with $S_{u}=6$ and $S_{v}=12$; the edge partition $E_{10}\left(D_{1}(n)\right)$ contains $4 n-4$ edges $u v$ with $S_{u}=6$ and $S_{v}=14$; the edge partition $E_{11}\left(D_{1}(n)\right)$ contains $4 n-4$ edges $u v$ with $S_{u}=7$ and $S_{v}=9$; the edge partition $E_{12}\left(D_{1}(n)\right)$ contains $4 n-4$ edges $u v$ with $S_{u}=7$ and $S_{v}=12$; the edge partition $E_{13}\left(D_{1}(n)\right)$ contains $12 n-8$ edges $u v$ with $S_{u}=8$ and $S_{v}=11$; the edge partition $E_{14}\left(D_{1}(n)\right)$ contains $4 n-4$ edges $u v$ with $S_{u}=8$ and $S_{v}=13$; the edge partition $E_{15}\left(D_{1}(n)\right)$ contains $2 n-2$ edges $u v$ with $S_{u}=S_{v}=9$; the edge partition $E_{16}\left(D_{1}(n)\right)$ contains $4 n-4$ edges $u v$ with $S_{u}=9$ and $S_{v}=14$; the edge partition $E_{17}\left(D_{1}(n)\right)$ contains $9 n^{2}-7 n+3$ edges $u v$ with $S_{u}=S_{v}=11 ;$ the edge partition $E_{18}\left(D_{1}(n)\right)$ contains 4 edges $u v$ with $S_{u}=11$ and $S_{v}=12$; the edge partition $E_{19}\left(D_{1}(n)\right)$ contains $4 n-4$ edges $u v$ with $S_{u}=11$ and $S_{v}=13$; the edge partition $E_{20}\left(D_{1}(n)\right)$ contains $36 n^{2}-68 n+32$ edges $u v$ with $S_{u}=11$ and $S_{v}=14$; the edge partition $E_{21}\left(D_{1}(n)\right)$ contains $4 n-4$ edges $u v$ with $S_{u}=11$ and $S_{v}=16$; the edge partition $E_{22}\left(D_{1}(n)\right)$ contains $4 n-4$ edges $u v$ with $S_{u}=12$ and $S_{v}=14$; the edge partition $E_{23}\left(D_{1}(n)\right)$ contains $4 n-4$ edges $u v$ with $S_{u}=13$ and $S_{v}=14$; the edge partition $E_{24}\left(D_{1}(n)\right)$ contains $4 n-4$ edges $u v$ with $S_{u}=13$ and $S_{v}=16$; the edge partition $E_{25}\left(D_{1}(n)\right)$ contains $4 n-4$ edges $u v$ with $S_{u}=S_{v}=14$ and the edge partition $E_{26}\left(D_{1}(n)\right)$ contains $36 n^{2}-76 n+40$ edges $u v$ with $S_{u}=14$ and $S_{v}=16$.

From equation (7), we get

$$
A B C_{4}(G)=\sum_{u v \in E(G)} \sqrt{\frac{S_{u}+S_{v}-2}{S_{u} S_{v}}}=\sum_{j=7}^{26} \sum_{u v \in E_{j}(G)} \sqrt{\frac{S_{u}+S_{v}-2}{S_{u} S_{v}}} .
$$

By using the edge partition given in Table 2, we get the following $A B C_{4}\left(D_{1}(n)\right)=\frac{\sqrt{10}}{6}\left|E_{7}\left(D_{1}(n)\right)\right|+\frac{\sqrt{110}}{22}\left|E_{8}\left(D_{1}(n)\right)\right|+\frac{\sqrt{2}}{3}\left|E_{9}\left(D_{1}(n)\right)\right|+\frac{\sqrt{42}}{14}\left|E_{10}\left(D_{1}(n)\right)\right|+\frac{\sqrt{2}}{3}\left|E_{11}\left(D_{1}(n)\right)\right|+$
$\frac{\sqrt{357}}{42}\left|E_{12}\left(D_{1}(n)\right)\right|+\frac{\sqrt{374}}{44}\left|E_{13}\left(D_{1}(n)\right)\right|+\frac{\sqrt{494}}{52}\left|E_{14}\left(D_{1}(n)\right)\right|+\frac{4}{9}\left|E_{15}\left(D_{1}(n)\right)\right|+\frac{\sqrt{6}}{6}\left|E_{16}\left(D_{1}(n)\right)\right|+$ 


\begin{tabular}{|c|c|c|c|}
\hline$\left(S_{u}, S_{v}\right)$ where $u v \in E(G)$ & Number of edges & $\left(S_{u}, S_{v}\right)$ where $u v \in E(G)$ & Number of edges \\
\hline$(6,6)$ & $4 n$ & $(11,11)$ & $9 n^{2}-7 n+3$ \\
\hline$(6,11)$ & $4 n$ & $(11,12)$ & 4 \\
\hline$(6,12)$ & 4 & $(11,13)$ & $4 n-4$ \\
\hline$(6,14)$ & $4 n-4$ & $(11,14)$ & $36 n^{2}-68 n+32$ \\
\hline$(7,9)$ & $4 n-4$ & $(11,16)$ & $4 n-4$ \\
\hline$(7,12)$ & $4 n-4$ & $(12,14)$ & $4 n-4$ \\
\hline$(8,11)$ & $12 n-8$ & $(13,14)$ & $4 n-4$ \\
\hline$(8,13)$ & $4 n-4$ & $(13,16)$ & $4 n-4$ \\
\hline$(9,9)$ & $2 n-2$ & $(14,14)$ & $4 n-4$ \\
\hline$(9,14)$ & $4 n-4$ & $(14,16)$ & $36 n^{2}-76 n+40$ \\
\hline
\end{tabular}

Table 2. Edge partition of Dominating David Derived network of first type $\left(D_{1}(n)\right)$ based on sum of degrees of end vertices of each edge.

$2 \frac{\sqrt{5}}{11}\left|E_{17}\left(D_{1}(n)\right)\right|+\frac{\sqrt{77}}{22}\left|E_{18}\left(D_{1}(n)\right)\right|+\frac{\sqrt{26}}{13}\left|E_{19}\left(D_{1}(n)\right)\right|+\frac{\sqrt{23}}{\sqrt{154}}\left|E_{20}\left(D_{1}(n)\right)\right|+5 \frac{\sqrt{11}}{44}\left|E_{21}\left(D_{1}(n)\right)\right|+$
$\frac{\sqrt{7}}{7}\left|E_{22}\left(D_{1}(n)\right)\right|+\frac{5}{\sqrt{182}}\left|E_{23}\left(D_{1}(n)\right)\right|+3 \frac{\sqrt{39}}{52}\left|E_{24}\left(D_{1}(n)\right)\right|+\frac{\sqrt{26}}{14}\left|E_{25}\left(D_{1}(n)\right)\right|+\frac{\sqrt{2}}{4}\left|E_{26}\left(D_{1}(n)\right)\right|$.

After some calculation, we get the following

$\Longrightarrow A B C_{4}\left(D_{1}(n)\right)=\left(\frac{63 \sqrt{20}+693 \sqrt{2}+18 \sqrt{3542}}{77}\right) n^{2}+\left(\frac{8}{9}-\frac{53}{3} \sqrt{2}+\frac{2}{3} \sqrt{6}+\frac{2}{7} \sqrt{26}+\frac{4}{13} \sqrt{26}+\frac{3}{13} \sqrt{39}+\frac{4}{\sqrt{7}}+\frac{5}{\sqrt{11}}+\right.$ $\left.\frac{2}{3} \sqrt{10}+\frac{2}{11} \sqrt{110}+\frac{2}{7} \sqrt{42}+\frac{2}{21} \sqrt{357}+\frac{3}{11} \sqrt{374}+\frac{\sqrt{494}}{13}-\frac{7}{11} \sqrt{20}-\frac{34}{77} \sqrt{3542}+\frac{20}{\sqrt{182}}\right) n+10 \sqrt{2}-\frac{2}{3} \sqrt{6}-\frac{3}{13} \sqrt{39}-$ $\frac{2}{7} \sqrt{42}-\frac{4}{\sqrt{7}}-\frac{5}{\sqrt{11}}-\frac{4}{13} \sqrt{26}+\frac{2}{11} \sqrt{77}-\frac{2}{21} \sqrt{357}-\frac{2}{11} \sqrt{374}-\frac{\sqrt{494}}{13}+\frac{16}{77} \sqrt{3542}-\frac{20}{\sqrt{182}}-\frac{8}{9}+\frac{3}{11} \sqrt{20}-\frac{2}{7} \sqrt{26}$ From equation(8), we get

$$
G A_{5}(G)=\sum_{u v \in E(G)} \frac{2 \sqrt{S_{u} S_{v}}}{S_{u}+S_{v}}=\sum_{j=7}^{26} \sum_{u v \in E_{j}(G)} \frac{2 \sqrt{S_{u} S_{v}}}{S_{u}+S_{v}}
$$

By using the edge partition given in Table 2, we get the following $G A_{5}\left(D_{1}(n)\right)=\left|E_{7}\left(D_{1}(n)\right)\right|+2 \frac{\sqrt{66}}{17}\left|E_{8}\left(D_{1}(n)\right)\right|+2 \frac{\sqrt{2}}{3}\left|E_{9}\left(D_{1}(n)\right)\right|+\frac{\sqrt{21}}{5}\left|E_{10}\left(D_{1}(n)\right)\right|+3 \frac{\sqrt{7}}{8}\left|E_{11}\left(D_{1}(n)\right)\right|+$ $4 \frac{\sqrt{21}}{19}\left|E_{12}\left(D_{1}(n)\right)\right|+4 \frac{\sqrt{22}}{19}\left|E_{13}\left(D_{1}(n)\right)\right|+4 \frac{\sqrt{26}}{21}\left|E_{14}\left(D_{1}(n)\right)\right|+\left|E_{15}\left(D_{1}(n)\right)\right|+6 \frac{\sqrt{14}}{23}\left|E_{16}\left(D_{1}(n)\right)\right|+\left|E_{17}\left(D_{1}(n)\right)\right|+$ $4 \frac{\sqrt{33}}{23}\left|E_{18}\left(D_{1}(n)\right)\right|+\frac{\sqrt{143}}{12}\left|E_{19}\left(D_{1}(n)\right)\right|+2 \frac{\sqrt{154}}{25}\left|E_{20}\left(D_{1}(n)\right)\right|+8 \frac{\sqrt{11}}{27}\left|E_{21}\left(D_{1}(n)\right)\right|+2 \frac{\sqrt{42}}{13}\left|E_{22}\left(D_{1}(n)\right)\right|+$ $2 \frac{\sqrt{182}}{27}\left|E_{23}\left(D_{1}(n)\right)\right|+8 \frac{\sqrt{13}}{29}\left|E_{24}\left(D_{1}(n)\right)\right|+\left|E_{25}\left(D_{1}(n)\right)\right|+4 \frac{\sqrt{14}}{15}\left|E_{26}\left(D_{1}(n)\right)\right|$.

After some calculations, we have

$\Longrightarrow G A_{5}\left(D_{1}(n)\right)=\left(\frac{225+240 \sqrt{14}+72 \sqrt{154}}{25}\right) n^{2}+\left(3+\frac{3}{2} \sqrt{7}+\frac{32}{27} \sqrt{11}+\frac{32}{29} \sqrt{13}-\frac{304}{15} \sqrt{14}+\frac{24}{23} \sqrt{14}+\frac{4}{5} \sqrt{21}+\right.$ $\left.\frac{16}{19} \sqrt{21}+\frac{48}{19} \sqrt{22}+\frac{16}{21} \sqrt{26}+\frac{8}{13} \sqrt{42}+\frac{8}{17} \sqrt{66}+\frac{\sqrt{143}}{3}+\frac{8}{27} \sqrt{182}-\frac{136}{25} \sqrt{154}\right) n+\frac{8}{3} \sqrt{2}-\frac{3}{2} \sqrt{7}-\frac{32}{27} \sqrt{11}-\frac{32}{29} \sqrt{13}+$ $\frac{32}{3} \sqrt{14}-\frac{24}{23} \sqrt{14}-\frac{4}{5} \sqrt{21}-\frac{16}{19} \sqrt{21}-\frac{32}{19} \sqrt{22}-\frac{16}{21} \sqrt{26}+\frac{16}{23} \sqrt{33}-\frac{8}{13} \sqrt{42}-\frac{\sqrt{143}}{3}+\frac{64}{25} \sqrt{154}-\frac{8}{27} \sqrt{182}-3$. 


\subsection{Results for Dominating David Derived networks of second type}

In this section, we calculate some degree based topological indices of Dominating David Derived network of second type denoted by $D_{2}(n)$ of dimension $n$. We compute the general Randić index $R_{\alpha}(G)$ with $\alpha=\left\{1,-1, \frac{1}{2},-\frac{1}{2}\right\}, A B C, G A, A B C_{4}$ and $G A_{5}$ for $D_{2}(n)$.

Theorem 2.2.1. Let $G \cong D_{2}(n)$ denotes the Dominating David Derived network of second type, then its general Randić index is equal to

$$
R_{\alpha}\left(D_{2}(n)\right)= \begin{cases}1116 n^{2}-1396 n+516, & \alpha=1 ; \\ (72 \sqrt{3}+18 \sqrt{6}+144) n^{2}+(56 \sqrt{2}-112 \sqrt{3}- & \\ 22 \sqrt{6}-200) n-32 \sqrt{2}+48 \sqrt{3}+6 \sqrt{6}+80, & \alpha=\frac{1}{2} \\ \frac{108 n^{2}-85 n+27}{12}, & \alpha=-1 ; \\ (6 \sqrt{3}+3 \sqrt{6}+9) n^{2}+\left(7 \sqrt{2}-\frac{28}{\sqrt{3}}-\frac{22}{\sqrt{6}}-11\right) n+ & \\ 5-4 \sqrt{2}+4 \sqrt{3}+\sqrt{6}, & \alpha=-\frac{1}{2} .\end{cases}
$$

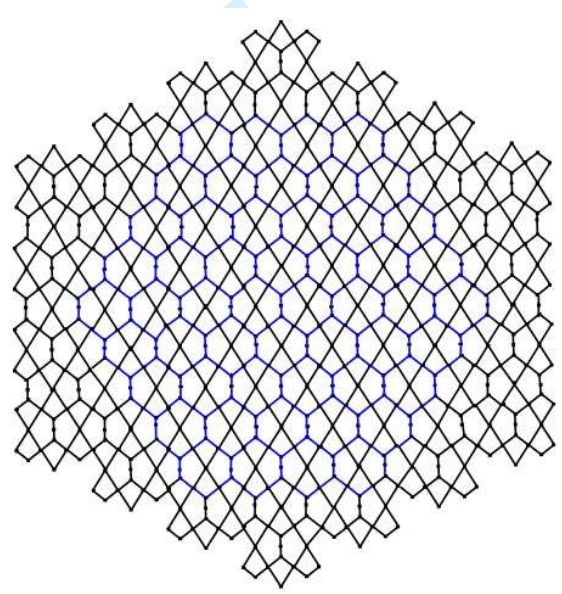

Fig. 2. Dominating David Derived network of second type $\left(D_{2}(4)\right)$

\begin{tabular}{|c|c|}
\hline$\left(d_{u}, d_{v}\right)$ where $u v \in E(G)$ & Number of edges \\
\hline$(2,2)$ & $4 n$ \\
\hline$(2,3)$ & $18 n^{2}-22 n+6$ \\
\hline$(2,4)$ & $28 n-16$ \\
\hline$(3,4)$ & $36 n^{2}-56 n+24$ \\
\hline$(4,4)$ & $36 n^{2}-52 n+20$ \\
\hline
\end{tabular}

Table 3. Edge partition of Dominating David Derived network of second type $\left(D_{2}(n)\right)$ based on degrees of end vertices of each edge.

Proof. Let $G$ be the Dominating David Derived network of second type. The second Dominating David Derived network denoted by $D_{2}(n)$ has $9 n^{2}+7 n-5$ vertices of degree $2,18 n^{2}-26 n+10$ vertices of 
degree 3 and $27 n^{2}-33 n+12$ vertices of degree 4 . The edge set of $D_{2}(n)$ is divided into five partitions based on the degree of end vertices. The first edge partition $E_{1}\left(D_{2}(n)\right)$ contains $4 n$ edges $u v$; where $\operatorname{deg}(u)=\operatorname{deg}(v)=2$. The second edge partition $E_{2}\left(D_{2}(n)\right)$ contains $18 n^{2}-22 n+6$ edges $u v$; where $\operatorname{deg}(u)=2$ and $\operatorname{deg}(v)=3$. The third edge partition $E_{3}\left(D_{2}(n)\right)$ contains $28 n-16$ edges $u v$; where $\operatorname{deg}(u)=2$ and $\operatorname{deg}(v)=4$. The fourth edge partition $E_{4}\left(D_{2}(n)\right)$ contains $36 n^{2}-56 n+24$ edges $u v ;$ where $\operatorname{deg}(u)=3$ and $\operatorname{deg}(v)=4$. The fifth edge partition $E_{5}\left(D_{2}(n)\right)$ contains $36 n^{2}-52 n+20$ edges $u v$; where $\operatorname{deg}(u)=\operatorname{deg}(v)=4$. Table 3 shows such an edge partition of Dominating David Derived network of second type $\left(D_{2}(n)\right)$. Thus from equation (3), it follows that

$$
R_{\alpha}(G)=\sum_{u v \in E(G)}(\operatorname{deg}(u) \cdot \operatorname{deg}(v))^{\alpha}
$$

For $\alpha=1$

Now we apply the formula of general Randić index $R_{\alpha}(G)$ for $\alpha=1$.

$$
R_{1}(G)=\sum_{j=1}^{5} \sum_{u v \in E_{j}(G)} \operatorname{deg}(u) \cdot \operatorname{deg}(v)
$$

By using the edge partition given in Table 3, we get

$$
\begin{gathered}
R_{1}(G)=4\left|E_{1}\left(D_{2}(n)\right)\right|+6\left|E_{2}\left(D_{2}(n)\right)\right|+8\left|E_{3}\left(D_{2}(n)\right)\right|+12\left|E_{4}\left(D_{2}(n)\right)\right|+16\left|E_{5}\left(D_{2}(n)\right)\right| . \\
\Longrightarrow R_{1}(G)=1116 n^{2}-1396 n+516 .
\end{gathered}
$$

For $\alpha=\frac{1}{2}$

We apply the formula of $R_{\alpha}(G)$ for $\alpha=\frac{1}{2}$.

$$
R_{\frac{1}{2}}(G)=\sum_{j=1}^{5} \sum_{u v \in E_{j}(G)} \sqrt{\operatorname{deg}(u) \cdot \operatorname{deg}(v)}
$$

By using the edge partition given in Table 3 , we get

$$
\begin{aligned}
& R_{\frac{1}{2}}\left(D_{2}(n)\right)=2\left|E_{1}\left(D_{2}(n)\right)\right|+\sqrt{6}\left|E_{2}\left(D_{2}(n)\right)\right|+2 \sqrt{2}\left|E_{3}\left(D_{2}(n)\right)\right|+2 \sqrt{3}\left|E_{4}\left(D_{2}(n)\right)\right|+4\left|E_{5}\left(D_{2}(n)\right)\right| . \\
& \Longrightarrow R_{\frac{1}{2}}\left(D_{2}(n)\right)=(72 \sqrt{3}+18 \sqrt{6}+144) n^{2}+(56 \sqrt{2}-112 \sqrt{3}-22 \sqrt{6}-200) n-32 \sqrt{2}+48 \sqrt{3}+6 \sqrt{6}+80
\end{aligned}
$$

For $\alpha=-1$

We apply the formula of $R_{\alpha}(G)$ for $\alpha=-1$.

$$
R_{-1}(G)=\sum_{j=1}^{5} \sum_{u v \in E_{j}(G)} \frac{1}{\operatorname{deg}(u) \cdot \operatorname{deg}(v)}
$$




$$
\begin{gathered}
R_{-1}\left(D_{2}(n)\right)=\frac{1}{4}\left|E_{1}\left(D_{2}(n)\right)\right|+\frac{1}{6}\left|E_{2}\left(D_{2}(n)\right)\right|+\frac{1}{8}\left|E_{3}\left(D_{2}(n)\right)\right|+\frac{1}{12}\left|E_{4}\left(D_{2}(n)\right)\right|+\frac{1}{16}\left|E_{5}\left(D_{2}(n)\right)\right| \\
\Longrightarrow R_{-1}\left(D_{2}(n)\right)=\frac{108 n^{2}-85 n+27}{12}
\end{gathered}
$$

For $\alpha=-\frac{1}{2}$

We apply the formula of $R_{\alpha}(G)$ for $\alpha=-\frac{1}{2}$.

$$
\begin{gathered}
R_{-\frac{1}{2}}(G)=\sum_{j=1}^{5} \sum_{u v \in E_{j}(G)} \frac{1}{\sqrt{\operatorname{deg}(u) \cdot \operatorname{deg}(v)}} \\
R_{-\frac{1}{2}}\left(D_{2}(n)\right)=\frac{1}{2}\left|E_{1}\left(D_{2}(n)\right)\right|+\frac{1}{\sqrt{6}}\left|E_{2}\left(D_{2}(n)\right)\right|+\frac{\sqrt{2}}{4}\left|E_{3}\left(D_{2}(n)\right)\right|+\frac{\sqrt{3}}{6}\left|E_{4}\left(D_{2}(n)\right)\right|+\frac{1}{4}\left|E_{5}\left(D_{2}(n)\right)\right| \\
\Longrightarrow R_{-\frac{1}{2}}\left(D_{2}(n)\right)=(6 \sqrt{3}+3 \sqrt{6}+9) n^{2}+\left(7 \sqrt{2}-\frac{28}{\sqrt{3}}-\frac{22}{\sqrt{6}}-11\right) n+5-4 \sqrt{2}+4 \sqrt{3}+\sqrt{6}
\end{gathered}
$$

In the following theorem, we compute the first Zagreb index of Dominating David Derived network of second type denoted by $D_{2}(n)$.

Theorem 2.2.2. For the Dominating David Derived network of second type $G \cong D_{2}(n)$, the first Zagreb index is equal to

$$
M_{1}\left(D_{2}(n)\right)=630 n^{2}-734 n+262
$$

Proof. Let $D_{2}(n)$ denotes the second Dominating David Derived network of second type. By using the edge partition from Table 3, the result follows. From equation (4), we have

$$
M_{1}(G)=\sum_{u v \in E(G)}(\operatorname{deg}(u)+\operatorname{deg}(v))=\sum_{j=1}^{5} \sum_{u v \in E_{j}(G)}(\operatorname{deg}(u)+\operatorname{deg}(v))
$$

$M_{1}\left(D_{2}(n)\right)=4\left|E_{1}\left(D_{2}(n)\right)\right|+5\left|E_{2}\left(D_{2}(n)\right)\right|+6\left|E_{3}\left(D_{2}(n)\right)\right|+7\left|E_{4}\left(D_{2}(n)\right)\right|+8\left|E_{5}\left(D_{2}(n)\right)\right|$

By doing the some calculation, we get the following

$$
\Longrightarrow M_{1}\left(D_{2}(n)\right)=630 n^{2}-734 n+262
$$

Next, we compute the $A B C, G A, A B C_{4}$ and $G A_{5}$ indices of Dominating David Derived network of second type denoted by $D_{2}(n)$.

Theorem 2.2.3. Let $G \cong D_{2}(n)$ be the Dominating David Derived network of second type, then we have - $A B C(G)=(9 \sqrt{2}+9 \sqrt{6}+6 \sqrt{15}) n^{2}+\left(5 \sqrt{2}-13 \sqrt{6}-\frac{28}{3} \sqrt{15}\right) n-5 \sqrt{2}+5 \sqrt{6}+4 \sqrt{15}$, for every positive integer $n \geq 1$.

- $G A(G)=\left(\frac{1260+720 \sqrt{3}+252 \sqrt{6}}{35}\right) n^{2}+\left(\frac{56}{3} \sqrt{2}-32 \sqrt{3}-\frac{44}{5} \sqrt{6}-48\right) n+20-\frac{32}{3} \sqrt{2}+\frac{96}{7} \sqrt{3}+\frac{12}{5} \sqrt{6}$, for every 
positive integer $n \geq 1$.

- $A B C_{4}(G)=\left(\frac{315 \sqrt{2}+21 \sqrt{210}+18 \sqrt{770}}{35}\right) n^{2}+\left(2-19 \sqrt{2}+\frac{2}{3} \sqrt{10}+\frac{5}{\sqrt{11}}+\frac{2}{7} \sqrt{26}+\frac{2}{7} \sqrt{35}+\frac{2}{7} \sqrt{42}+\frac{4}{\sqrt{7}}+\right.$ $\frac{2}{11} \sqrt{110}+\frac{\sqrt{182}}{7}+\frac{20}{\sqrt{182}}-\sqrt{210}+\frac{351}{\sqrt{13}}+\frac{2}{21} \sqrt{357}+\frac{3}{11} \sqrt{374}+\frac{\sqrt{494}}{13}-\frac{36}{35} \sqrt{770}-\frac{4}{55} \sqrt{2090}+\frac{2}{65} \sqrt{2730}+$ $\left.\frac{2}{77} \sqrt{3542}\right) n+\frac{34}{3} \sqrt{2}-\frac{2}{7} \sqrt{26}-\frac{2}{7} \sqrt{35}-\frac{2}{7} \sqrt{42}-\frac{4}{\sqrt{7}}+\frac{2}{11} \sqrt{77}-\frac{5}{\sqrt{11}}-\frac{20}{\sqrt{182}}-\frac{\sqrt{182}}{7}+\frac{7}{15} \sqrt{210}-\frac{\sqrt{351}}{13}-$ $\frac{2}{21} \sqrt{357}-\frac{2}{11} \sqrt{374}-\frac{\sqrt{494}}{13}+\frac{18}{35} \sqrt{770}-\frac{2}{55} \sqrt{2090}-\frac{2}{65} \sqrt{2730}-\frac{2}{77} \sqrt{3542}-2$, for every positive integer $n \geq 2$.

- $G A_{5}(G)=\left(\frac{96 \sqrt{14}+45 \sqrt{15}+60 \sqrt{35}}{10}\right) n^{2}+\left(8+\frac{16}{7} \sqrt{3}+\frac{16}{11} \sqrt{7}+\frac{32}{27} \sqrt{11}+\frac{32}{29} \sqrt{13}+\frac{16}{15} \sqrt{14}-\frac{96}{13} \sqrt{14}-\frac{15}{2} \sqrt{15}+\right.$ $\left.\frac{4}{5} \sqrt{21}+\frac{16}{19} \sqrt{21}+\frac{48}{19} \sqrt{22}+\frac{16}{21} \sqrt{26}-12 \sqrt{35}+\frac{8}{13} \sqrt{42}+\frac{8}{17} \sqrt{66}+\frac{8}{11} \sqrt{110}+\frac{8}{23} \sqrt{130}+\frac{8}{25} \sqrt{154}+\frac{8}{27} \sqrt{182}\right) n+$ $\frac{8}{3} \sqrt{2}-\frac{16}{7} \sqrt{3}-\frac{16}{11} \sqrt{7}-\frac{32}{27} \sqrt{11}-\frac{32}{29} \sqrt{13}+\frac{32}{3} \sqrt{14}-\frac{16}{15} \sqrt{14}+\frac{7}{2} \sqrt{15}-\frac{4}{5} \sqrt{21}-\frac{16}{19} \sqrt{21}-\frac{32}{19} \sqrt{22}-\frac{16}{21} \sqrt{26}+$ $\frac{16}{23} \sqrt{33}+6 \sqrt{35}-\frac{8}{13} \sqrt{42}-\frac{4}{11} \sqrt{110}-\frac{8}{23} \sqrt{130}-\frac{8}{25} \sqrt{154}-\frac{8}{27} \sqrt{182}-4$, for every positive integer $n \geq 2$.

\begin{tabular}{|c|c|c|c|}
\hline$\left(S_{u}, S_{v}\right)$ where $u v \in E(G)$ & Number of edges & $\left(S_{u}, S_{v}\right)$ where $u v \in E(G)$ & Number of edges \\
\hline$(6,6)$ & $4 n$ & $(10,11)$ & $8 n-4$ \\
\hline$(6,8)$ & $4 n-4$ & $(10,13)$ & $4 n-4$ \\
\hline$(6,10)$ & $18 n^{2}-30 n+14$ & $(10,14)$ & $36 n^{2}-72 n+36$ \\
\hline$(6,11)$ & $4 n$ & $(11,12)$ & 4 \\
\hline$(6,12)$ & 4 & $(11,14)$ & $4 n-4$ \\
\hline$(6,14)$ & $4 n-4$ & $(11,16)$ & $4 n-4$ \\
\hline$(7,8)$ & $4 n-4$ & $(12,14)$ & $4 n-4$ \\
\hline$(7,12)$ & $4 n-4$ & $(13,14)$ & $4 n-4$ \\
\hline$(8,11)$ & $12 n-8$ & $(13,16)$ & $4 n-4$ \\
\hline$(8,13)$ & $4 n-4$ & $(14,14)$ & $36 n^{2}-76 n+40$ \\
\hline$(8,14)$ & $4 n-4$ & $(14,16)$ & \\
\hline
\end{tabular}

Table 4. Edge partition of Dominating David Derived network of second type $\left(D_{2}(n)\right)$ based on sum of degrees of end vertices of each edge.

Proof. By using the edge partition given in Table 3, we get the result. From equation (5), it follows that

$$
A B C(G)=\sum_{u v \in E(G)} \sqrt{\frac{\operatorname{deg}(u)+\operatorname{deg}(v)-2}{\operatorname{deg}(u) \cdot \operatorname{deg}(v)}}=\sum_{j=1}^{5} \sum_{u v \in E_{j}(G)} \sqrt{\frac{\operatorname{deg}(u)+\operatorname{deg}(v)-2}{\operatorname{deg}(u) \cdot \operatorname{deg}(v)}} .
$$

$A B C\left(D_{2}(n)\right)=\frac{1}{\sqrt{2}}\left|E_{1}\left(D_{2}(n)\right)\right|+\frac{1}{\sqrt{2}}\left|E_{2}\left(D_{2}(n)\right)\right|+\frac{1}{\sqrt{2}}\left|E_{3}\left(D_{2}(n)\right)\right|+\frac{\sqrt{15}}{6}\left|E_{4}\left(D_{2}(n)\right)\right|+\frac{\sqrt{6}}{4}\left|E_{5}\left(D_{2}(n)\right)\right|$.

By doing the some calculation, we get

$\Longrightarrow A B C\left(D_{2}(n)\right)=(9 \sqrt{2}+9 \sqrt{6}+6 \sqrt{15}) n^{2}+\left(5 \sqrt{2}-13 \sqrt{6}-\frac{28}{3} \sqrt{15}\right) n-5 \sqrt{2}+5 \sqrt{6}+4 \sqrt{15}$

From equation(6), we get

$$
G A\left(D_{2}(n)\right)=\sum_{u v \in E(G)} \frac{2 \sqrt{\operatorname{deg}(u) \cdot \operatorname{deg}(v)}}{\operatorname{deg}(u)+\operatorname{deg}(v)}=\sum_{j=1}^{5} \sum_{u v \in E_{j}(G)} \frac{2 \sqrt{\operatorname{deg}(u) \cdot \operatorname{deg}(v)}}{\operatorname{deg}(u)+\operatorname{deg}(v)} .
$$


By using the edge partition given in Table 3, we get

$G A\left(D_{2}(n)\right)=\left|E_{1}\left(D_{2}(n)\right)\right|+2 \frac{\sqrt{6}}{5}\left|E_{2}\left(D_{2}(n)\right)\right|+2 \frac{\sqrt{2}}{6}\left|E_{3}\left(D_{2}(n)\right)\right|+4 \frac{\sqrt{3}}{7}\left|E_{4}\left(D_{2}(n)\right)\right|+\left|E_{5}\left(D_{2}(n)\right)\right|$.

By doing the some calculation, we get

$\Longrightarrow G A\left(D_{2}(n)\right)=\left(\frac{1260+720 \sqrt{3}+252 \sqrt{6}}{35}\right) n^{2}+\left(\frac{56}{3} \sqrt{2}-32 \sqrt{3}-\frac{44}{5} \sqrt{6}-48\right) n+20-\frac{32}{3} \sqrt{2}+\frac{96}{7} \sqrt{3}+\frac{12}{5} \sqrt{6}$.

If we consider edge partitions based on degree sum of neighbors of end vertices, then the edge set $E\left(D_{2}(n)\right)$ can be divided into twenty two edge partition $E_{j}\left(D_{2}(n)\right), 6 \leq j \leq 27$; where the edge partition $E_{6}\left(D_{2}(n)\right)$ contains $4 n$ edges $u v$ with $S_{u}=S_{v}=6$. The edge partition $E_{7}\left(D_{2}(n)\right)$ contains $4 n-4$ edges $u v$ with $S_{u}=6$ and $S_{v}=8$, the edge partition $E_{8}\left(D_{2}(n)\right)$ contains $18 n^{2}-30 n+14$ edges $u v$ with $S_{u}=6$ and $S_{v}=10$, the edge partition $E_{9}\left(D_{2}(n)\right)$ contains $4 n$ edges $u v$ with $S_{u}=6$ and $S_{v}=11$; the edge partition $E_{10}\left(D_{2}(n)\right)$ contains 4 edges $u v$ with $S_{u}=6$ and $S_{v}=12$; the edge partition $E_{11}\left(D_{2}(n)\right)$ contains $4 n-4$ edges $u v$ with $S_{u}=6$ and $S_{v}=14$; the edge partition $E_{12}\left(D_{2}(n)\right)$ contains $4 n-4$ edges $u v$ with $S_{u}=7$ and $S_{v}=8$; the edge partition $E_{13}\left(D_{2}(n)\right)$ contains $4 n-4$ edges $u v$ with $S_{u}=7$ and $S_{v}=12$; the edge partition $E_{14}\left(D_{2}(n)\right)$ contains $12 n-8$ edges $u v$ with $S_{u}=8$ and $S_{v}=11$; the edge partition $E_{15}\left(D_{2}(n)\right)$ contains $4 n-4$ edges $u v$ with $S_{u}=8$ and $S_{v}=13$, the edge partition $E_{16}\left(D_{2}(n)\right)$ contains $4 n-4$ edges $u v$ with $S_{u}=8$ and $S_{v}=14$, the edge partition $E_{17}\left(D_{2}(n)\right)$ contains $8 n-4$ edges $u v$ with $S_{u}=10$ and $S_{v}=11$; the edge partition $E_{18}\left(D_{2}(n)\right)$ contains $4 n-4$ edges $u v$ with $S_{u}=10$ and $S_{v}=13$; the edge partition $E_{19}\left(D_{2}(n)\right)$ contains $36 n^{2}-72 n+36$ edges $u v$ with $S_{u}=10$ and $S_{v}=14$, the edge partition $E_{20}\left(D_{2}(n)\right)$ contains 4 edges $u v$ with $S_{u}=11$ and $S_{v}=12$, the edge partition $E_{21}\left(D_{2}(n)\right)$ contains $4 n-4$ edges $u v$ with $S_{u}=11$ and $S_{v}=14$; the edge partition $E_{22}\left(D_{2}(n)\right)$ contains $4 n-4$ edges $u v$ with $S_{u}=11$ and $S_{v}=16$; the edge partition $E_{23}\left(D_{2}(n)\right)$ contains $4 n-4$ edges $u v$ with $S_{u}=12$ and $S_{v}=14$; the edge partition $E_{24}\left(D_{2}(n)\right)$ contains $4 n-4$ edges $u v$ with $S_{u}=13$ and $S_{v}=14$; the edge partition $E_{25}\left(D_{2}(n)\right)$ contains $4 n-4$ edges $u v$ with $S_{u}=13$ and $S_{v}=16$; the edge partition $E_{26}\left(D_{2}(n)\right)$ contains $4 n-4$ edges $u v$ with $S_{u}=S_{v}=14$ and the edge partition $E_{27}\left(D_{2}(n)\right)$ contains $36 n^{2}-76 n+40$ edges $u v$ with $S_{u}=14$ and $S_{v}=16$.

From equation (7), we get

$$
A B C_{4}(G)=\sum_{u v \in E(G)} \sqrt{\frac{S_{u}+S_{v}-2}{S_{u} S_{v}}}=\sum_{j=6}^{27} \sum_{u v \in E_{j}(G)} \sqrt{\frac{S_{u}+S_{v}-2}{S_{u} S_{v}}} .
$$

By using the edge partition given in Table 4, we get the following

$$
\begin{aligned}
& A B C_{4}\left(D_{2}(n)\right)=\frac{\sqrt{10}}{6}\left|E_{6}\left(D_{2}(n)\right)\right|+\frac{1}{2}\left|E_{7}\left(D_{2}(n)\right)\right|+\frac{\sqrt{210}}{30}\left|E_{8}\left(D_{2}(n)\right)\right|+\frac{\sqrt{110}}{22}\left|E_{9}\left(D_{2}(n)\right)\right|+\frac{\sqrt{2}}{3}\left|E_{10}\left(D_{2}(n)\right)\right|+ \\
& \frac{\sqrt{42}}{14}\left|E_{11}\left(D_{2}(n)\right)\right|+\frac{\sqrt{182}}{28}\left|E_{12}\left(D_{2}(n)\right)\right|+\frac{\sqrt{357}}{42}\left|E_{13}\left(D_{2}(n)\right)\right|+\frac{\sqrt{374}}{44}\left|E_{14}\left(D_{2}(n)\right)\right|+\frac{\sqrt{494}}{52}\left|E_{15}\left(D_{2}(n)\right)\right|+ \\
& \frac{\sqrt{35}}{14}\left|E_{16}\left(D_{2}(n)\right)\right|+\sqrt{\frac{19}{110}}\left|E_{17}\left(D_{2}(n)\right)\right|+\sqrt{\frac{21}{130}}\left|E_{18}\left(D_{2}(n)\right)\right|+\frac{\sqrt{770}}{70}\left|E_{19}\left(D_{2}(n)\right)\right|+\frac{\sqrt{77}}{22}\left|E_{20}\left(D_{2}(n)\right)\right|+
\end{aligned}
$$


$\sqrt{\frac{23}{154}}\left|E_{21}\left(D_{2}(n)\right)\right|+5 \frac{\sqrt{11}}{44}\left|E_{22}\left(D_{2}(n)\right)\right|+\frac{\sqrt{7}}{7}\left|E_{23}\left(D_{2}(n)\right)\right|+\frac{5}{\sqrt{182}}\left|E_{24}\left(D_{2}(n)\right)\right|+3 \frac{\sqrt{39}}{52}\left|E_{25}\left(D_{2}(n)\right)\right|+$ $\frac{\sqrt{26}}{14}\left|E_{26}\left(D_{2}(n)\right)\right|+\frac{\sqrt{2}}{4}\left|E_{27}\left(D_{2}(n)\right)\right|$.

After some calculation, we get the following

$\Longrightarrow A B C_{4}\left(D_{2}(n)\right)=\left(\frac{315 \sqrt{2}+21 \sqrt{210}+18 \sqrt{770}}{35}\right) n^{2}+\left(2-19 \sqrt{2}+\frac{2}{3} \sqrt{10}+\frac{5}{\sqrt{11}}+\frac{2}{7} \sqrt{26}+\frac{2}{7} \sqrt{35}+\frac{2}{7} \sqrt{42}+\right.$ $\frac{4}{\sqrt{7}}+\frac{2}{11} \sqrt{110}+\frac{\sqrt{182}}{7}+\frac{20}{\sqrt{182}}-\sqrt{210}+\frac{351}{\sqrt{13}}+\frac{2}{21} \sqrt{357}+\frac{3}{11} \sqrt{374}+\frac{\sqrt{494}}{13}-\frac{36}{35} \sqrt{770}-\frac{4}{55} \sqrt{2090}+\frac{2}{65} \sqrt{2730}+$ $\left.\frac{2}{77} \sqrt{3542}\right) n+\frac{34}{3} \sqrt{2}-\frac{2}{7} \sqrt{26}-\frac{2}{7} \sqrt{35}-\frac{2}{7} \sqrt{42}-\frac{4}{\sqrt{7}}+\frac{2}{11} \sqrt{77}-\frac{5}{\sqrt{11}}-\frac{20}{\sqrt{182}}-\frac{\sqrt{182}}{7}+\frac{7}{15} \sqrt{210}-\frac{\sqrt{351}}{13}-$ $\frac{2}{21} \sqrt{357}-\frac{2}{11} \sqrt{374}-\frac{\sqrt{494}}{13}+\frac{18}{35} \sqrt{770}-\frac{2}{55} \sqrt{2090}-\frac{2}{65} \sqrt{2730}-\frac{2}{77} \sqrt{3542}-2$.

From equation (8), we get

$$
G A_{5}(G)=\sum_{u v \in E(G)} \frac{2 \sqrt{S_{u} S_{v}}}{S_{u}+S_{v}}=\sum_{j=6}^{27} \sum_{u v \in E_{j}(G)} \frac{2 \sqrt{S_{u} S_{v}}}{S_{u}+S_{v}}
$$

By using the edge partition given in Table 4, we get the following

$$
\begin{aligned}
& G A_{5}\left(D_{2}(n)\right)=\left|E_{6}\left(D_{2}(n)\right)\right|+4 \frac{\sqrt{3}}{7}\left|E_{7}\left(D_{2}(n)\right)\right|+\frac{\sqrt{15}}{4}\left|E_{8}\left(D_{2}(n)\right)\right|+2 \frac{\sqrt{66}}{17}\left|E_{9}\left(D_{2}(n)\right)\right|+2 \frac{\sqrt{2}}{3}\left|E_{10}\left(D_{2}(n)\right)\right|+ \\
& \frac{\sqrt{21}}{5}\left|E_{11}\left(D_{2}(n)\right)\right|+4 \frac{\sqrt{14}}{15}\left|E_{12}\left(D_{2}(n)\right)\right|+4 \frac{\sqrt{21}}{19}\left|E_{13}\left(D_{2}(n)\right)\right|+4 \frac{\sqrt{22}}{19}\left|E_{14}\left(D_{2}(n)\right)\right|+4 \frac{\sqrt{26}}{21}\left|E_{15}\left(D_{2}(n)\right)\right|+ \\
& 4 \frac{\sqrt{7}}{11}\left|E_{16}\left(D_{2}(n)\right)\right|+2 \frac{\sqrt{110}}{21}\left|E_{17}\left(D_{2}(n)\right)\right|+2 \frac{\sqrt{130}}{23}\left|E_{18}\left(D_{2}(n)\right)\right|+\frac{\sqrt{35}}{6}\left|E_{19}\left(D_{2}(n)\right)\right|+4 \frac{\sqrt{33}}{23}\left|E_{20}\left(D_{2}(n)\right)\right|+ \\
& 2 \frac{\sqrt{154}}{25}\left|E_{21}\left(D_{2}(n)\right)\right|+8 \frac{\sqrt{11}}{27}\left|E_{22}\left(D_{2}(n)\right)\right|+2 \frac{\sqrt{42}}{13}\left|E_{23}\left(D_{2}(n)\right)\right|+2 \frac{\sqrt{182}}{27}\left|E_{24}\left(D_{2}(n)\right)\right|+8 \frac{\sqrt{13}}{29}\left|E_{25}\left(D_{2}(n)\right)\right|+ \\
& \left|E_{26}\left(D_{2}(n)\right)\right|+4 \frac{\sqrt{14}}{15}\left|E_{27}\left(D_{2}(n)\right)\right| .
\end{aligned}
$$

After calculation, we get

$\Longrightarrow G A_{5}\left(D_{2}(n)\right)=\left(\frac{96 \sqrt{14}+45 \sqrt{15}+60 \sqrt{35}}{10}\right) n^{2}+\left(8+\frac{16}{7} \sqrt{3}+\frac{16}{11} \sqrt{7}+\frac{32}{27} \sqrt{11}+\frac{32}{29} \sqrt{13}+\frac{16}{15} \sqrt{14}-\frac{96}{13} \sqrt{14}-\right.$ $\frac{15}{2} \sqrt{15}+\frac{4}{5} \sqrt{21}+\frac{16}{19} \sqrt{21}+\frac{48}{19} \sqrt{22}+\frac{16}{21} \sqrt{26}-12 \sqrt{35}+\frac{8}{13} \sqrt{42}+\frac{8}{17} \sqrt{66}+\frac{8}{11} \sqrt{110}+\frac{8}{23} \sqrt{130}+\frac{8}{25} \sqrt{154}+$ $\left.\frac{8}{27} \sqrt{182}\right) n+\frac{8}{3} \sqrt{2}-\frac{16}{7} \sqrt{3}-\frac{16}{11} \sqrt{7}-\frac{32}{27} \sqrt{11}-\frac{32}{29} \sqrt{13}+\frac{32}{3} \sqrt{14}-\frac{16}{15} \sqrt{14}+\frac{7}{2} \sqrt{15}-\frac{4}{5} \sqrt{21}-\frac{16}{19} \sqrt{21}-\frac{32}{19} \sqrt{22}-$ $\frac{16}{21} \sqrt{26}+\frac{16}{23} \sqrt{33}+6 \sqrt{35}-\frac{8}{13} \sqrt{42}-\frac{4}{11} \sqrt{110}-\frac{8}{23} \sqrt{130}-\frac{8}{25} \sqrt{154}-\frac{8}{27} \sqrt{182}-4$.

\subsection{Results for Dominating David Derived networks of third type}

We calculate certain degree based topological indices of Dominating David Derived network of third type denoted by $D_{3}(n)$ (of dimension $n$ ) in this section. We compute the general Randić index $R_{\alpha}(G)$ with $\alpha=\left\{1,-1, \frac{1}{2},-\frac{1}{2}\right\}, A B C, G A, A B C_{4}$ and $G A_{5}$ in the following theorems for $D_{3}(n)$.

The general Randić index for $D_{3}(n)$ is computed in the following theorem. 
Theorem 2.3.1. Let $G \cong D_{3}(n)$ denotes the Dominating David Derived network of third type, then its general Randić index is equal to

$$
R_{\alpha}\left(D_{3}(n)\right)= \begin{cases}1440 n^{2}-1872 n+704, & \alpha=1 ; \\ (288+72 \sqrt{2}) n^{2}-(40 \sqrt{2}+424) n+176, & \alpha=\frac{1}{2} \\ \frac{36 n^{2}-33 n+11}{4}, & \alpha=-1 \\ (18+9 \sqrt{2}) n^{2}-(25+5 \sqrt{2}) n+44, & \alpha=-\frac{1}{2}\end{cases}
$$

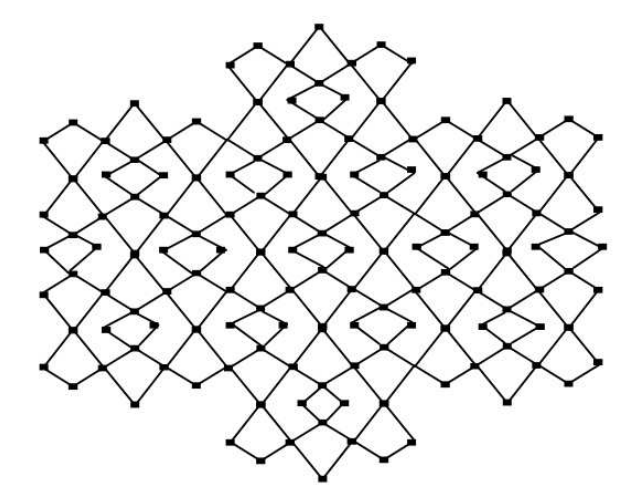

Fig. 3. Dominating David Derived network of third type type $\left(D_{3}(2)\right)$

\begin{tabular}{|c|c|}
\hline$\left(d_{u}, d_{v}\right)$ where $u v \in E(G)$ & Number of edges \\
\hline$(2,2)$ & $4 n$ \\
\hline$(2,4)$ & $36 n^{2}-20 n$ \\
\hline$(4,4)$ & $72 n^{2}-108 n+44$ \\
\hline
\end{tabular}

Table 5. Edge partition of Dominating David Derived network of third type $\left(D_{3}(n)\right)$ based on degrees of end vertices of each edge.

Proof. Let $D_{3}(n)$ be the Dominating David Derived network of third type. The Dominating David Derived network of third type denoted by $D_{3}(n)$ has $18 n^{2}-6 n$ vertices of degree 2 and $45 n^{2}-59 n+22$ vertices of degree 4 . The edge set of $D_{3}(n)$ is divided into three partitions based on the degree of end vertices. The first edge partition $E_{1}\left(D_{3}(n)\right)$ contains $4 n$ edges $u v$; where $\operatorname{deg}(u)=\operatorname{deg}(v)=2$. The second edge partition $E_{2}\left(D_{3}(n)\right)$ contains $36 n^{2}-20 n$ edges $u v$; where $\operatorname{deg}(u)=2$ and $\operatorname{deg}(v)=4$. The third edge partition $E_{3}\left(D_{3}(n)\right)$ contains $72 n^{2}-108 n+44$ edges $u v$; where $\operatorname{deg}(u)=\operatorname{deg}(v)=4$. Table 5 shows such an edge partition of $D_{3}(n)$. Thus from equation (3), is follows that

$$
R_{\alpha}(G)=\sum_{u v \in E(G)}(\operatorname{deg}(u) \cdot \operatorname{deg}(v))^{\alpha}
$$


For $\alpha=1$

Now we apply the formula of $R_{\alpha}(G)$ for $\alpha=1$.

$$
R_{1}(G)=\sum_{j=1}^{3} \sum_{u v \in E_{j}(G)} \operatorname{deg}(u) \cdot \operatorname{deg}(v)
$$

By using the edge partition given in Table 5, we get

$$
\begin{gathered}
R_{1}(G)=4\left|E_{1}\left(D_{3}(n)\right)\right|+8\left|E_{2}\left(D_{3}(n)\right)\right|+16\left|E_{3}\left(D_{3}(n)\right)\right| \\
\Longrightarrow R_{1}(G)=1440 n^{2}-1872 n+704
\end{gathered}
$$

For $\alpha=\frac{1}{2}$

We apply the formula of $R_{\alpha}(G)$ for $\alpha=\frac{1}{2}$.

$$
R_{\frac{1}{2}}(G)=\sum_{j=1}^{3} \sum_{u v \in E_{j}(G)} \sqrt{\operatorname{deg}(u) \cdot \operatorname{deg}(v)}
$$

By using the edge partition given in Table 5, we get

$$
\begin{aligned}
& R_{\frac{1}{2}}(G)=2\left|E_{1}\left(D_{3}(n)\right)\right|+2 \sqrt{2}\left|E_{2}\left(D_{3}(n)\right)\right|+4\left|E_{3}\left(D_{3}(n)\right)\right| \\
& \Longrightarrow R_{\frac{1}{2}}(G)=(288+72 \sqrt{2}) n^{2}-(40 \sqrt{2}+424) n+176
\end{aligned}
$$

For $\alpha=-1$

We apply the formula of $R_{\alpha}(G)$ for $\alpha=-1$.

$$
\begin{gathered}
R_{-1}(G)=\sum_{j=1}^{3} \sum_{u v \in E_{j}(G)} \frac{1}{\operatorname{deg}(u) \cdot \operatorname{deg}(v)} \\
R_{-1}(G)=\frac{1}{4}\left|E_{1}\left(D_{3}(n)\right)\right|+\frac{1}{8}\left|E_{2}\left(D_{3}(n)\right)\right|+\frac{1}{16}\left|E_{3}\left(D_{3}(n)\right)\right| \\
\Longrightarrow R_{-1}(G)=\frac{36 n^{2}-33 n+11}{4}
\end{gathered}
$$

For $\alpha=-\frac{1}{2}$ 
We apply the formula of $R_{\alpha}(G)$ for $\alpha=-\frac{1}{2}$.

$$
\begin{gathered}
R_{-\frac{1}{2}}(G)=\sum_{j=1}^{3} \sum_{u v \in E_{j}(G)} \frac{1}{\sqrt{\operatorname{deg}(u) \cdot \operatorname{deg}(v)}} \\
R_{-\frac{1}{2}}(G)=\frac{1}{2}\left|E_{1}\left(D_{3}(n)\right)\right|+\frac{\sqrt{2}}{4}\left|E_{2}\left(D_{3}(n)\right)\right|+\frac{1}{4}\left|E_{3}\left(D_{3}(n)\right)\right| \\
\Longrightarrow R_{-\frac{1}{2}}(G)=(18+9 \sqrt{2}) n^{2}-(25+5 \sqrt{2}) n+44
\end{gathered}
$$

In the following theorem, we compute the first Zagreb index of Dominating David Derived network of third type denoted by $D_{3}(n)$.

Theorem 2.3.2. For Dominating David Derived network of third type denoted by $G \cong D_{3}(n)$, the first Zagreb index is equal to

$$
M_{1}\left(D_{3}(n)\right)=792 n^{2}-968 n+352
$$

Proof. Let $D_{3}(n)$ denotes the Dominating David Derived network of third type. By using the edge partition from Table 5, the result follows. From equation(4) we have

$$
\begin{gathered}
M_{1}(G)=\sum_{u v \in E(G)}(\operatorname{deg}(u)+\operatorname{deg}(v))=\sum_{j=1}^{3} \sum_{u v \in E_{j}(G)}(\operatorname{deg}(u)+\operatorname{deg}(v)) \\
M_{1}(G)=4\left|E_{1}\left(D_{2}(n)\right)\right|+6\left|E_{2}\left(D_{3}(n)\right)\right|+8\left|E_{3}\left(D_{3}(n)\right)\right|
\end{gathered}
$$

By doing some calculation, we get

$$
\Longrightarrow M_{1}(G)=792 n^{2}-968 n+352
$$

Next, we compute the $A B C, G A, A B C_{4}$ and $G A_{5}$ indices for Dominating David Derived network of third type denoted by $D_{3}(n)$.

Theorem 2.3.3. Let $G \cong D_{3}(n)$ be the Dominating David Derived network of third type, then we have

- $A B C(G)=(18 \sqrt{2}+18 \sqrt{6}) n^{2}-(8 \sqrt{2}+27 \sqrt{6}) n+11 \sqrt{6}$, for every positive integer $n \geq 1$.

- $G A(G)=(72+24 \sqrt{2}) n^{2}-\left(\frac{40}{3} \sqrt{2}+104\right) n+44$, for every positive integer $n \geq 1$.

- $A B C_{4}(G)=\left(\frac{32 \sqrt{3}+9 \sqrt{30}+6 \sqrt{78}}{4}\right) n^{2}+\left(\frac{7}{3} \sqrt{2}-11 \sqrt{3}+\frac{2}{3} \sqrt{10}+\frac{2}{5} \sqrt{15}\right.$

$\left.+\frac{6}{5} \sqrt{20}+\frac{2}{3} \sqrt{22}+\frac{2}{7} \sqrt{26}-\frac{19}{4} \sqrt{30}+\frac{2}{7} \sqrt{35}+\frac{2}{7} \sqrt{42}+\frac{8}{\sqrt{7}}-\frac{5}{2} \sqrt{78}\right) n$

$+\frac{7}{3} \sqrt{2}+4 \sqrt{3}-\frac{2}{5} \sqrt{15}-\frac{6}{5} \sqrt{20}-\frac{2}{7} \sqrt{26}+\frac{5}{2} \sqrt{30}-\frac{2}{7} \sqrt{35}-\frac{2}{7} \sqrt{42}+\frac{2}{3} \sqrt{78}$, for every positive integer $n \geq 2$.

- $G A_{5}(G)=\left(\frac{1260+720 \sqrt{3}+504 \sqrt{6}}{35}\right) n^{2}+\left(\frac{8}{3} \sqrt{2}-\frac{240}{17} \sqrt{3}+\frac{16}{3} \sqrt{5}-\frac{88}{5} \sqrt{6}+\frac{16}{11} \sqrt{7}+\frac{16}{13} \sqrt{10}+\frac{16}{15} \sqrt{14}+\frac{4}{5} \sqrt{21}+\right.$ $\left.\frac{16}{13} \sqrt{42}-60\right) n+\frac{8}{3} \sqrt{2}+\frac{64}{7} \sqrt{3}-\frac{16}{3} \sqrt{5}+\frac{32}{5} \sqrt{6}-\frac{16}{11} \sqrt{7}-\frac{16}{13} \sqrt{10}+\frac{16}{15} \sqrt{14}-\frac{4}{5} \sqrt{21}-\frac{16}{13} \sqrt{42}+36$, for every positive integer $n \geq 2$. 


\begin{tabular}{|c|c|c|c|}
\hline$\left(S_{u}, S_{v}\right)$ where $u v \in E(G)$ & Number of edges & $\left(S_{u}, S_{v}\right)$ where $u v \in E(G)$ & Number of edges \\
\hline$(6,6)$ & $4 n$ & $(12,12)$ & $8 n$ \\
\hline$(6,12)$ & $4 n+4$ & $(12,14)$ & $8 n-8$ \\
\hline$(6,14)$ & $4 n-4$ & $(12,16)$ & $36 n^{2}-60 n+16$ \\
\hline$(8,10)$ & $12 n-12$ & $(14,14)$ & $4 n-4$ \\
\hline$(8,12)$ & $36 n^{2}-44 n+16$ & $(14,16)$ & $4 n+4$ \\
\hline$(8,14)$ & $4 n-4$ & $(16,16)$ & $36 n^{2}-76 n+40$ \\
\hline$(10,16)$ & $4 n-4$ & & \\
\hline
\end{tabular}

Table 6. Edge partition of Dominating David Derived network of third $\operatorname{type}\left(D_{3}(n)\right)$ based on sum of degrees of end vertices of each edge.

Proof. By using the edge partition given in Table 5, we get the result. From equation (5), it follows that

$$
\begin{gathered}
A B C(G)=\sum_{u v \in E(G)} \sqrt{\frac{\operatorname{deg}(u)+\operatorname{deg}(v)-2}{\operatorname{deg}(u) \cdot \operatorname{deg}(v)}}=\sum_{j=1}^{3} \sum_{u v \in E_{j}(G)} \sqrt{\frac{\operatorname{deg}(u)+\operatorname{deg}(v)-2}{\operatorname{deg}(u) \cdot \operatorname{deg}(v)} .} \\
A B C\left(D_{3}(n)\right)=\frac{1}{\sqrt{2}}\left|E_{1}\left(D_{3}(n)\right)\right|+\frac{1}{\sqrt{2}}\left|E_{2}\left(D_{3}(n)\right)\right|+\frac{\sqrt{6}}{4}\left|E_{3}\left(D_{3}(n)\right)\right| .
\end{gathered}
$$

By doing some calculation, we get

$$
\Longrightarrow A B C(G)=(18 \sqrt{2}+18 \sqrt{6}) n^{2}-(8 \sqrt{2}+27 \sqrt{6}) n+11 \sqrt{6}
$$

From equation (6), we get

$$
G A(G)=\sum_{u v \in E(G)} \frac{2 \sqrt{\operatorname{deg}(u) \cdot \operatorname{deg}(v)}}{\operatorname{deg}(u)+\operatorname{deg}(v)}=\sum_{j=1}^{3} \sum_{u v \in E_{j}(G)} \frac{2 \sqrt{\operatorname{deg}(u) \cdot \operatorname{deg}(v)}}{\operatorname{deg}(u)+\operatorname{deg}(v)}
$$

By doing some calculation, we get

$$
G A\left(D_{3}(n)\right)=\left|E_{1}\left(D_{3}(n)\right)\right|+2 \frac{\sqrt{2}}{3}\left|E_{2}\left(D_{3}(n)\right)\right|+\left|E_{3}\left(D_{3}(n)\right)\right| .
$$

By doing some calculation, we get

$$
\Longrightarrow G A=(72+24 \sqrt{2}) n^{2}-\left(\frac{40}{3} \sqrt{2}+104\right) n+44 .
$$

If we consider edge partitions based on degree sum of neighbors of end vertices, then the edge set $E\left(D_{3}(n)\right)$ can be divided into thirteen edge partition $E_{j}\left(D_{2}(n)\right), 4 \leq j \leq 16$; where the edge partition $E_{4}\left(D_{3}(n)\right)$ contains $4 n$ edges $u v$ with $S_{u}=S_{v}=6$; the edge partition $E_{5}\left(D_{3}(n)\right)$ contains $4 n+4$ edges $u v$ with $S_{u}=6$ and $S_{v}=12$; the edge partition $E_{6}\left(D_{3}(n)\right)$ contains $4 n-4$ edges $u v$ with $S_{u}=6$ and $S_{v}=14$; the edge partition $E_{7}\left(D_{3}(n)\right)$ contains $12 n-12$ edges $u v$ with $S_{u}=8$ and $S_{v}=10$, the edge partition $E_{8}\left(D_{3}(n)\right)$ contains $36 n^{2}-44 n+16$ edges $u v$ with $S_{u}=8$ and $S_{v}=12$, the edge partition $E_{9}\left(D_{3}(n)\right)$ contains $4 n-4$ edges $u v$ with $S_{u}=8$ and $S_{v}=14$; the edge partition $E_{10}\left(D_{3}(n)\right)$ contains 
$4 n-4$ edges $u v$ with $S_{u}=10$ and $S_{v}=16$; the edge partition $E_{11}\left(D_{3}(n)\right)$ contains $8 n$ edges $u v$ with $S_{u}=S_{v}=12$; the edge partition $E_{12}\left(D_{3}(n)\right)$ contains $8 n-8$ edges $u v$ with $S_{u}=12$ and $S_{v}=14$; the edge partition $E_{13}\left(D_{3}(n)\right)$ contains $36 n^{2}-60 n+16$ edges $u v$ with $S_{u}=12$ and $S_{v}=16$; the edge partition $E_{14}\left(D_{3}(n)\right)$ contains $4 n-4$ edges $u v$ with $S_{u}=S_{v}=14$; the edge partition $E_{15}\left(D_{3}(n)\right)$ contains $4 n+4$ edges $u v$ with $S_{u}=14$ and $S_{v}=16$; and the edge partition $E_{16}\left(D_{3}(n)\right)$ contains $36 n^{2}-76 n+40$ edges $u v$ with $S_{u}=S_{v}=16$.

From equation (7), we get

$$
A B C_{4}(G)=\sum_{u v \in E(G)} \sqrt{\frac{S_{u}+S_{v}-2}{S_{u} S_{v}}}=\sum_{j=4}^{16} \sum_{u v \in E_{j}(G)} \sqrt{\frac{S_{u}+S_{v}-2}{S_{u} S_{v}}} .
$$

By using the edge partition given in Table 6 , we get the following

$$
\begin{aligned}
& A B C_{4}\left(D_{3}(n)\right)=\frac{\sqrt{10}}{6}\left|E_{4}\left(D_{3}(n)\right)\right|+\frac{\sqrt{2}}{3}\left|E_{5}\left(D_{3}(n)\right)\right|+\frac{\sqrt{42}}{14}\left|E_{6}\left(D_{3}(n)\right)\right|+\frac{\sqrt{5}}{5}\left|E_{7}\left(D_{3}(n)\right)\right|+\frac{\sqrt{3}}{4}\left|E_{8}\left(D_{3}(n)\right)\right|+ \\
& \frac{\sqrt{35}}{14}\left|E_{9}\left(D_{3}(n)\right)\right|+\frac{\sqrt{15}}{10}\left|E_{10}\left(D_{3}(n)\right)\right|+\frac{\sqrt{22}}{12}\left|E_{11}\left(D_{3}(n)\right)\right|+\frac{\sqrt{7}}{7}\left|E_{12}\left(D_{3}(n)\right)\right|+\frac{\sqrt{78}}{24}\left|E_{13}\left(D_{3}(n)\right)\right|+\frac{\sqrt{26}}{14}\left|E_{14}\left(D_{3}(n)\right)\right|+ \\
& \frac{\sqrt{2}}{4}\left|E_{15}\left(D_{3}(n)\right)\right|+\frac{\sqrt{30}}{16}\left|E_{16}\left(D_{3}(n)\right)\right| .
\end{aligned}
$$

After some calculation, we get

$\Longrightarrow A B C_{4}\left(D_{3}(n)\right)=\left(\frac{32 \sqrt{3}+9 \sqrt{30}+6 \sqrt{78}}{4}\right) n^{2}+\left(\frac{7}{3} \sqrt{2}-11 \sqrt{3}+\frac{2}{3} \sqrt{10}+\frac{2}{5} \sqrt{15}+\frac{6}{5} \sqrt{20}+\frac{2}{3} \sqrt{22}+\frac{2}{7} \sqrt{26}-\right.$
$\left.\frac{19}{4} \sqrt{30}+\frac{2}{7} \sqrt{35}+\frac{2}{7} \sqrt{42}+\frac{8}{\sqrt{7}}-\frac{5}{2} \sqrt{78}\right) n+\frac{7}{3} \sqrt{2}+4 \sqrt{3}-\frac{2}{5} \sqrt{15}-\frac{6}{5} \sqrt{20}-\frac{2}{7} \sqrt{26}+\frac{5}{2} \sqrt{30}-\frac{2}{7} \sqrt{35}-\frac{2}{7} \sqrt{42}+\frac{2}{3} \sqrt{78}$.

From equation (8), we get

$$
G A_{5}(G)=\sum_{u v \in E(G)} \frac{2 \sqrt{S_{u} S_{v}}}{S_{u}+S_{v}}=\sum_{j=4}^{16} \sum_{u v \in E_{j}(G)} \frac{2 \sqrt{S_{u} S_{v}}}{S_{u}+S_{v}} .
$$

By using the edge partition given in Table 6 , we have

$$
\begin{aligned}
& \left.G A_{5}\left(D_{3}(n)\right)\right)=\left|E_{4}\left(D_{3}(n)\right)\right|+2 \frac{\sqrt{2}}{3}\left|E_{5}\left(D_{3}(n)\right)\right|+\frac{\sqrt{21}}{5}\left|E_{6}\left(D_{3}(n)\right)\right|+4 \frac{\sqrt{5}}{9}\left|E_{7}\left(D_{3}(n)\right)\right|+2 \frac{\sqrt{6}}{5}\left|E_{8}\left(D_{3}(n)\right)\right|+ \\
& 4 \frac{\sqrt{7}}{11}\left|E_{9}\left(D_{3}(n)\right)\right|+4 \frac{\sqrt{10}}{13}\left|E_{10}\left(D_{3}(n)\right)\right|+\left|E_{11}\left(D_{3}(n)\right)\right|+2 \frac{\sqrt{42}}{13}\left|E_{12}\left(D_{3}(n)\right)\right|+4 \frac{\sqrt{3}}{7}\left|E_{13}\left(D_{3}(n)\right)\right|+\left|E_{14}\left(D_{3}(n)\right)\right|+ \\
& 4 \frac{\sqrt{14}}{15}\left|E_{15}\left(D_{3}(n)\right)\right|+\left|E_{16}\left(D_{3}(n)\right)\right| .
\end{aligned}
$$

After calculation, we get

$\left.\Longrightarrow G A_{5}\left(D_{3}(n)\right)\right)=\left(\frac{1260+720 \sqrt{3}+504 \sqrt{6}}{35}\right) n^{2}+\left(\frac{8}{3} \sqrt{2}-\frac{240}{17} \sqrt{3}+\frac{16}{3} \sqrt{5}-\frac{88}{5} \sqrt{6}+\frac{16}{11} \sqrt{7}+\frac{16}{13} \sqrt{10}+\frac{16}{15} \sqrt{14}+\right.$ $\left.\frac{4}{5} \sqrt{21}+\frac{16}{13} \sqrt{42}-60\right) n+\frac{8}{3} \sqrt{2}+\frac{64}{7} \sqrt{3}-\frac{16}{3} \sqrt{5}+\frac{32}{5} \sqrt{6}-\frac{16}{11} \sqrt{7}-\frac{16}{13} \sqrt{10}+\frac{16}{15} \sqrt{14}-\frac{4}{5} \sqrt{21}-\frac{16}{13} \sqrt{42}+36$. 


\section{Conclusion}

In this paper, certain degree based topological indices, namely general Randić index, atom-bond connectivity index $(A B C)$, geometric-arithmetic index $(G A)$ and first Zagreb index for Dominating David Derived networks of first, second and third type were studied for the first time and analytical closed formulas for these networks were determined which will help the people working in network science to understand and explore the underlying topologies of these networks.

In future, we are interested to design some new architectures/networks and then study their topological indices which will be quite helpful to understand their underlying topologies.

\section{References}

1. Wiener H. J. Am. Chem. Soc. 1947,69, 17.

2. Deza, M.; Fowler, P. W.; Rassat, A.; Rogers, K. M. J. Chem. Inf. Comput. Sci. 2000, 40, 550.

3. Star of David [online] available, http://en.wikipedia.org/wiki/Star of David.

4. Simonraj, F.; George, A. GRAPH-HOC 2012, 4, 11.

5. Diudea, M. V.; Gutman, I.; Lorentz, J. Molecular Topology; Nova Science Publishers: Hauppauge, NY, 2001.

6. Gutman, I.; Polansky, O. E. Mathematical concepts in organic chemistry, Springer-Verlag, New York, 1986.

7. Randić, M. J. Amer. Chem. Soc. 1975,97, 6609.

8. Estrada, E.; Torres, L.; Rodríguez, L.; Gutman, I. Indian J. Chem. 1998, 37A, 849.

9. Vukičević, D.; Furtula, B. J. Math. Chem. 2009, 46, 1369.

10. Ghorbani, M.; Hosseinzadeh, M. A. Optoelectron. Adv. Mater. Rapid Commun. 2010, 4, 1419.

11. Graovac, A.; Ghorbani, M.; Hosseinzadeh, M. A. J. Math. Nanosci. 2011, 1, 33.

12. Hayat, S.; Imran, M. Appl. Math. Comput. 2014, 240, 213.

13. Bača, M.; Horváthová, J.; Mokrišová, M.; Suhányiovă, A. Appl. Math. Comput. 2015, 251, 154.

14. Baig, A. Q.; Imran, M.; Ali, H. Optoelectron. Adv. Mater. Rapid Commun. 2015, 9, 248.

15. Baig, A. Q.; Imran, M.; Ali, H. Canad. J. Chem. 2015, 93, 730.

16. Hayat, S.; Imran, M. J. Comput. Theor. Nanosci. 2015, 12, 70.

17. Hayat, S.; Imran, M. J. Comput. Theor. Nanosci. 2015, 12, 533.

18. Hayat, S.; Imran, M. Appl. Math. Comput. 2014, 240, 213.

19. Iranmanesh, A.; Zeraatkar, M. Optoelectron. Adv. Mater. Rapid Commun. 2010, 4, 1852.

20. Lin, W.; Chen, J.; Chen, Q.; Gao, T.; Lin, X.; Cai, B. MATCH Commun. Math. Comput. Chem.2014,72, 699.

21. Manuel, P. D.; Abd-El-Barr, M. I.; Rajasingh, I.; Rajan, B. J. Discrete Algorithms 2008, 6, 11.

22. Palacios, J.L. MATCH Commun. Math. Comput. Chem. 2014, 72, 709.

23. Simonraj, F.; George, A. Int. J. Future Comput. Commun., 2013,2, 90. 


\section{Figure Captions}

Fig. 1. Dominating David Derived network of first type $\left(D_{1}(2)\right)$

Fig. 2. Dominating David Derived network of second type $\left(D_{2}(4)\right)$

Fig. 3. Dominating David Derived network of third type type $\left(D_{3}(2)\right)$ 